\title{
Evaporative cooling at low trap depth
}

\section{Citation}

deCarvalho, Robert, and John Doyle. 2004. "Evaporative Cooling at Low Trap Depth." Physical Review A 70 (5) (November 18). doi:10.1103/physreva.70.053409.

\section{Published Version}

doi:10.1103/PhysRevA.70.053409

\section{Permanent link}

http://nrs.harvard.edu/urn-3:HUL.InstRepos:28425790

\section{Terms of Use}

This article was downloaded from Harvard University's DASH repository, and is made available under the terms and conditions applicable to Other Posted Material, as set forth at http:// nrs.harvard.edu/urn-3:HUL.InstRepos:dash.current.terms-of-use\#LAA

\section{Share Your Story}

The Harvard community has made this article openly available.

Please share how this access benefits you. Submit a story.

\section{Accessibility}




\title{
Evaporative cooling at low trap depth
}

\author{
Robert deCarvalho and John Doyle \\ Harvard University, Cambridge, Massachusetts 02138, USA \\ (Received 13 February 2004; published 18 November 2004)
}

\begin{abstract}
A quantitative, analytic model of evaporative cooling covering both the small- $(<4)$ and large- $(>4) \eta$ regimes is presented. $\eta$ is the dimensionless parameter defined as the trap depth divided by the temperature of the trapped sample. Although some of the same general properties present at large $\eta$ are also present at small $\eta$, there are significant quantitative differences. These differences must be taken into account in order to accurately extract from the trapping data quantitative measurements of, for example, collisional atomic cross sections.
\end{abstract}

DOI: 10.1103/PhysRevA.70.053409

PACS number(s): 32.80.Pj, 32.80.Cy, 31.15.Bs

\section{INTRODUCTION}

The technique of evaporative cooling has so far proved to be a necessity for the production of dilute quantum gases. Although the experimental implementations are quite diverse, in each successful realization of quantum degenerate gases, evaporative cooling has been employed in the final cooling stage.

Over the last decade, buffer gas loading of magnetic traps has proved to be a versatile technique for loading large numbers of arbitrary paramagnetic species into magnetic traps [1-5]. Evaporative cooling of buffer gas loaded atoms has led to the observation of intriguing scattering behavior in the classical-to-quantum transition region characterized by scattering in a few partial waves. Extremely large inelastic scattering rates between $\mathrm{Cr}$ atoms were observed [6,7]. The largeness of this spin-changing inelastic rate, in comparison with the associated elastic scattering rate, seriously impacted the evaporative cooling efficiency. Not only was trap loss increased, but inelastic collisions heated the atoms to temperatures approaching the magnetic trap depth. Only at these elevated temperatures was evaporative cooling able to counteract the inelastic heating. This resulted in increasingly small values of $\eta \equiv$ (trap depth)/(trapped atom temperature). In analyzing these experimental results (e.g., extracting the inelastic $\mathrm{Cr}-\mathrm{Cr}$ scattering cross section), it was found that evaporative cooling at low $\eta$ 's lacked quantitative theory. This paper corrects the situation.

The literature contains various treatments that describe systems of evaporating atoms in the presence of additional loss mechanisms [8-10]. In general, the dynamics of these systems is quite difficult to model analytically. Simplifying approximations are often employed to provide insight into the behavior of such systems. The most common of these is the "large- $\eta$ " approximation. The large- $\eta$ model is discussed at length in the treatments by Doyle [9] and Ketterle [10] and is implicit in the treatment by Luiten [8]. However, since this model is not valid at low $\eta$, it is incapable of describing the above mentioned experimental observations. Indeed, any experiment that traverses the low- $\eta$ regime $(\eta<4)$ requires a quantitative model to accurately interpret the measured quantities.

This paper presents an alternate model that is an exact analytic treatment for all $\eta$, with the only "approximation" being the assumption of a spherically symmetric trapping potential. Although this model is not rigorously correct for the ellipsoidal trapping potential of a spherical quadrupole trap, it offers excellent quantitative results and provides a great deal of insight into the dynamics of magnetically confined atoms. It should be noted that a similar treatment could be used to analytically determine the behavior of any system having cylindrical symmetry. For simplicity, however, we restrict the scope of this paper to the special case of a spherically symmetric trap whose potential varies linearly with the radius.

\section{THE "LARGE- $\eta$ " MODEL}

In this section, we review the approach commonly used to describe evaporation dynamics in the case of large $\eta$. The large- $\eta$ approximation involves modeling the spatial profile of the atom cloud as if it obeyed a Boltzmann distribution abruptly truncated at the trap wall. In any given differential volume, the kinetic energy of the atoms is also assumed to obey a Boltzmann distribution truncated at a value equal to the energy required to escape the trap from the differential volume considered. Since the atom density is suppressed at the trap edge by $e^{-\eta}$, any distortions created by the trap edge become less important as $\eta$ is increased.

\section{A. Large- $\boldsymbol{\eta}$ effective volumes}

In many calculations involving ensembles of trapped atoms, the size of the atom cloud is an important parameter. A useful definition for the size of the cloud is the effective volume. The effective volume is defined such that the total number of atoms is given by the peak density in the trap multiplied by the effective volume, or $N=n_{0} V_{\text {eff }}$. From this, it is straightforward to show that, in the large- $\eta$ approximation of the linear spherical trap,

$$
V_{e f f}=3 V_{0} \int_{0}^{1} e^{-\eta \rho} \rho^{2} d \rho \underset{\eta \gtrless 4}{\simeq} \frac{6 V_{0}}{\eta^{3}},
$$

where $\rho \equiv r / R, R$ is the trap radius, and $V_{0}=(4 / 3) \pi R^{3}$.

It is also useful to introduce a collisional effective volume

$$
\Lambda_{e f f}=3 V_{0} \int_{0}^{1}\left[\frac{n(\rho)}{n_{o}}\right]^{2} \rho^{2} d \rho \underset{\eta \gtrless 4}{\simeq} \frac{3 V_{0}}{4 \eta^{3}} .
$$


The collisional effective volume is needed for calculating the atom loss due to inelastic collisions. If each inelastic collision causes the loss of one atom, the peak density in the trap obeys the differential equation

$$
\left\langle\dot{n}_{0}\right\rangle=\frac{\Lambda_{\text {eff }}}{V_{\text {eff }}} n_{0} \sigma_{\text {in }}(\bar{v} \sqrt{2}) \underset{\eta \gtrless 4}{\simeq} \frac{1}{8} n_{0}^{2} \sigma_{\text {in }}(\bar{v} \sqrt{2}),
$$

where $\sigma_{i n}$ is the inelastic scattering cross section.

\section{B. Large- $\boldsymbol{\eta}$ evaporation fraction}

The fraction of elastic collisions that result in an atom with enough energy to leave the trap is called the evaporation fraction and can be calculated for large $\eta$ using the detailed balance model described by Ketterle [10].

This model starts off considering an ideal thermal gas confined in an infinite box potential. For a Boltzmann distribution, the fraction of atoms with kinetic energy $E_{K}$ greater than some arbitrary threshold value, which we will call $\eta k T$, is simply

$$
F=\frac{\int_{\eta k T}^{\infty} \sqrt{E_{K}} \exp \left(-E_{K} / k T\right) d E_{K}}{\int_{0}^{\infty} \sqrt{E_{K}} \exp \left(-E_{K} / k T\right) d E_{K}} \underset{\eta \gtrless 4}{\longrightarrow} 2 \sqrt{\frac{\eta}{\pi}} e^{-\eta} .
$$

For the infinite potential considered, an atom with energy greater than the arbitrary threshold $\eta k T$ would remain confined. If $\eta$ is large, there is a high probability that even a single collision will knock this atom to an energy lower than $\eta k T$. In order to maintain a steady state temperature, a different atom must experience collisions that promote it to energy greater than $\eta k T$. Thus, the rate at which atoms are promoted to energies greater than some threshold $\eta k T$ is the same as the collision rate experienced by the energetic $\left(E_{K}\right.$ $\geqslant \eta k T)$ atoms. This rate is just $\Gamma_{\text {thresh }}=n \sigma_{e l} v_{\eta} N_{\text {thresh }}$, where $n$ is the atom density in the infinite box potential, $\sigma_{e l}$ is the elastic scattering cross section, $v_{\eta}=\frac{1}{2} \bar{v} \sqrt{\pi \eta}$ is the velocity of atoms with energy $\eta k T, \bar{v}=\sqrt{8 k T / \pi m}$ is the mean thermal velocity, and $N_{\text {thresh }}=F N$ is the number of atoms above the threshold energy.

Although constructed for the case of an infinitely deep trap, this detailed balance between energy upscattering and downscattering remains true in the case of a finite trap with depth $\eta k T$. In this case, however, the upscattered atoms will be ejected from the trap before they can be downscattered. This allows us to write an expression for the evaporation rate from a box potential of finite depth $\eta k T$ :

$$
\Gamma_{b o x}=n \sigma_{e l} v_{\eta} F N=n \sigma_{e l} \bar{v} \eta e^{-\eta} N,
$$

where $N$ is the number of trapped atoms.

We can now adapt this box potential argument to create an evaporation model for the spherical linear trap. In such a trap, the kinetic energy required to evaporate an atom from some radius $r$ is equal to the difference in potential energy experienced by the atom in going from the radius $r$ to the trap radius, or $E_{\text {Kthresh }}=U(R)-U(r)$. This kinetic energy threshold for atom ejection can be used to define a position- dependent $\eta$ for evaporation from within a differential volume at a given radius $r$ in the trap, $\tilde{\eta}(r)=[U(R)$ $-U(r)] /\left(k_{B} T\right)$. Recalling that $\rho \equiv r / R, V_{0}=(4 / 3) \pi R^{3}$ and letting

$$
d N=n(r) d^{3} r=3 V_{0} n_{0} e^{-\eta \rho} \rho^{2} d \rho,
$$

Eq. (5) can be used to write the radially dependent differential evaporation rate as

$$
d \Gamma_{v}(\rho)=n(\rho) \sigma_{e l} \bar{v} \tilde{\eta} e^{-\tilde{\eta}} d N=3 V_{0} n_{0}^{2} \sigma_{e l} \bar{v} \eta e^{-\eta}(1-\rho) e^{-\eta \rho} \rho^{2} d \rho .
$$

To find the evaporation fraction, we must now calculate the elastic collision rate corresponding to this evaporation rate. The rate at which one atom undergoes collisions in the trap is given by $n \sigma_{e l} \bar{v} \sqrt{2}$ (the mean relative velocity between atoms is $\bar{v} \sqrt{2}$ ). The total collision rate for $d N$ atoms found at a radius $r$ in the trap is then just

$$
d \Gamma_{e l}(\rho)=n(\rho) \sigma_{e l} \bar{v} \sqrt{2} d N=3 V_{0} n_{0}^{2} \sigma_{e l} \bar{v} \sqrt{2} e^{-2 \eta \rho} \rho^{2} d \rho .
$$

As a function of radius, the fraction of elastic collisions resulting in evaporation is then given by

$$
\tilde{f}(\rho) \equiv \frac{d \Gamma_{v}(\rho)}{d \Gamma_{e l}(\rho)}=\frac{1}{\sqrt{2}} \eta(1-\rho) \exp [-\eta(1-\rho)] .
$$

To calculate the mean evaporation fraction for the trapped ensemble, we must average this collision rate over the entire volume of the trap. Using the normalized radial probability distribution for collisions in the trap,

$$
P_{c}(\rho) d \rho=\frac{n^{2}(\rho) \rho^{2} d \rho}{\int_{0}^{1} n^{2}(\rho) \rho^{2} d \rho}=\frac{4 \eta^{3} \rho^{2} e^{-2 \eta \rho}}{1-\left(2 \eta^{2}+2 \eta+1\right) e^{-2 \eta}},
$$

the average evaporation fraction for the trapped ensemble is

$f=\int_{0}^{1} P_{c}(\rho) \tilde{f}(\rho) d \rho=\frac{2 \sqrt{2}\left[e^{-\eta}(2 \eta-6)+e^{-2 \eta}\left(\eta^{2}+4 \eta+6\right)\right]}{1-\left(2 \eta^{2}+2 \eta+1\right) e^{-2 \eta}}$.

\section{Average energies at infinite $\eta$}

Following the treatment of Luiten et al. [8] and appropriately truncating the distributions, the energy density of states $g(E)$ can be calculated by integrating the momentum and position coordinates over all permitted phase space in the trap and counting only states that have energies equal to $E$,

$$
\begin{aligned}
g(E) & =\frac{1}{h^{3}} \int_{r=0}^{E / G} d^{3} r \int_{p=0}^{\sqrt{2 m(E-G r)}} \delta\left(E-G r-\frac{p^{2}}{2 m}\right) d^{3} p \\
& =\frac{256 \pi^{2} \sqrt{2 m^{3}}}{105(h G)^{3}} E^{7 / 2},
\end{aligned}
$$

where $h$ is Planck's constant. Using this density of states and integrating to $E \rightarrow \infty$ for an infinitely deep trap, the probabil- 
ity of finding an atom with energy between $E$ and $E+d E$ is

$$
\begin{aligned}
P(E) d E & =\frac{g(E) \exp (-E / k T) d E}{\int_{0}^{\infty} g(E) \exp (-E / k T) d E} \\
& =\frac{16}{105 \sqrt{\pi}} \frac{E^{7 / 2}}{(k T)^{9 / 2}} \exp \left(-\frac{E}{k T}\right) d E .
\end{aligned}
$$

Using Eq. (13), the mean energy of atoms in an infinite linear spherical trap is

$$
\bar{E}_{\infty}=\frac{9}{2} k T
$$

Noting that collisions are two-body processes which scale with the square of the density, the average potential energy of collisionally lost atoms from an infinite trap can be written as

$$
\bar{U}_{d_{\infty}}=\frac{\int_{0}^{\infty} U(r) n^{2}(r) r^{2} d r}{\int_{0}^{\infty} n^{2}(r) r^{2} d r}=\frac{G \int_{0}^{\infty} \exp (-2 G r / k T) r^{3} d r}{\int_{0}^{\infty} \exp (-2 G r / k T) r^{2} d r}=\frac{3}{2} k T .
$$

The average kinetic energy of collisionally lost atoms is given by

$$
\frac{\bar{K}_{d_{\infty}}}{k T}=\frac{\int_{0}^{\infty} \int_{0}^{\infty} \varepsilon_{1} \sqrt{\varepsilon_{1} \varepsilon_{2}} \sqrt{\varepsilon_{1}+\varepsilon_{2}} e^{-\left(\varepsilon_{1}+\varepsilon_{2}\right)} d \varepsilon_{1} d \varepsilon_{2}}{\int_{0}^{\infty} \int_{0}^{\infty} \sqrt{\varepsilon_{1} \varepsilon_{2}} \sqrt{\varepsilon_{1}+\varepsilon_{2}} e^{-\left(\varepsilon_{1}+\varepsilon_{2}\right)} d \varepsilon_{1} d \varepsilon_{2}}=7 / 4
$$

where $\varepsilon_{i}=E_{i} / k T, \sqrt{\varepsilon_{1} \varepsilon_{2}}$ represents the free particle density of states, and $\sqrt{\varepsilon_{1}+\varepsilon_{2}}$ represents the collisional relative velocity between two atoms. The average energy of collisionally lost atoms is then given by

$$
\bar{E}_{d_{\infty}}=\bar{K}_{d_{\infty}}+\bar{U}_{d_{\infty}}=(3.25) k T .
$$

The average evaporation energy from a quasi-infinite trap is calculated by finding the population fraction with energy above the threshold value, $\eta k T$. This gives

$$
\bar{E}_{v_{\infty}}=\frac{\int_{\eta k T}^{\infty} E_{K}^{9 / 2} \exp \left(-E_{K} / k T\right) d E_{K}}{\int_{\eta k T}^{\infty} E_{K}^{7 / 2} \exp \left(-E_{K} / k T\right) d E_{K}} \underset{\eta \rightarrow \infty}{\rightarrow \rightarrow+1) k T .}
$$

\section{Energies at large $\eta$}

For the case of large but finite $\eta$, we must consider the impact of the trap wall in depleting the atoms' spatial distribution for radii approaching that of the wall. We account for this depletion by artificially truncating the spatial distribution at a radius smaller than that of the trap wall. If we set $\chi$ $\equiv r_{\text {truncation }} / R<1$, we can calculate the mean potential energy in the trap as

$$
\frac{\bar{U}(\eta)}{k T}=\frac{\int_{0}^{\chi} \eta \rho e^{-\eta \rho} \rho^{2} d \rho}{\int_{0}^{\chi} e^{-\eta \rho} \rho^{2} d \rho}=3+\frac{(\chi \eta)^{3}}{2-2 e^{\chi \eta}+\chi \eta(2+\chi \eta)}
$$

where $\rho \equiv r / R$. We find that setting $\chi=0.85$ results in good agreement with the results obtained in the exact solution to the linear spherical trap developed below.

For calculating kinetic energies, it is useful to introduce the concept of an effective $\eta$ defined as

$$
\eta_{e f f} \equiv 1.035 \eta\left(1-\rho_{e f f}\right)
$$

where $\rho_{\text {eff }} \equiv\left(V_{e f f} / V_{0}\right)^{1 / 3}=6^{1 / 3} / \eta$. It can be shown that the maximum allowed energy in a spherical linear trap is $(3 / 2) \eta k T$. Atoms with energy greater than $\eta k T$ cause an increase in the effective $\eta$. By fitting to the result exact treatment of the spherical linear system, we find that a $3.5 \%$ increase in $\eta$ nicely accounts for the effect of these energetic atoms. The factor of $\left(1-\rho_{e f f}\right)$ arises from assuming that kinetic energy calculations for atoms at the effective radius are representative of the ensemble as a whole. The kinetic energy required for atoms to escape from this radius is reduced by a factor of $\left(1-\rho_{e f f}\right)$. Under these assumptions, the average kinetic energy is

$$
\frac{\bar{K}(\eta)}{k T}=\frac{\int_{0}^{\eta_{e f f}} \varepsilon \sqrt{\varepsilon} e^{-\varepsilon} d \varepsilon}{\int_{0}^{\eta_{e f f}} \sqrt{\varepsilon} e^{-\varepsilon} d \varepsilon}=\frac{3}{2}+\frac{\eta_{e f f}}{1-\frac{1}{2} \sqrt{\pi / \eta_{e f f}} \operatorname{erf}\left(\sqrt{\eta_{e f f}}\right) e^{\eta_{e f f}}} .
$$

The mean energy in the trap is then given by

$$
\bar{E}(\eta)=\bar{K}(\eta)+\bar{U}(\eta)
$$

and is plotted in Fig. 5 below.

The mean kinetic energy of collisionally lost atoms can be calculated by first considering the mean kinetic energy at the center of the trap, $\bar{K}_{0}$, and neglecting for now the corrections embodied in $\eta_{\text {eff. }}$. We obtain

$$
\frac{\bar{K}_{0}}{k T}=\frac{\int_{0}^{\eta} \varepsilon^{3 / 2} \exp [-\varepsilon] d \varepsilon}{\int_{0}^{\eta} \varepsilon^{1 / 2} \exp [-\varepsilon] d \varepsilon}=\frac{3}{2}+\frac{2 \eta^{3 / 2}}{2 \sqrt{\eta}-e^{\eta} \sqrt{\pi} \operatorname{erf}(\sqrt{\eta})}
$$

Compared to its value for infinitely large $\eta$, the fractional kinetic energy at the center of the trap is 


$$
\frac{\bar{K}_{0}}{\bar{K}_{0_{\infty}}}=1+\left(\frac{4}{3}\right) \frac{\eta^{3 / 2}}{2 \sqrt{\eta}-e^{\eta} \sqrt{\pi} \operatorname{erf}(\sqrt{\eta})} .
$$

We can scale the infinite trap collisional kinetic energy, Eq. (16), by the $\eta$-dependent kinetic energy fraction of Eq. (24) to obtain an expression for the mean energy of collisionally lost atoms as a function of $\eta$. Inserting the $\eta_{\text {eff }}$ corrections, we obtain

$$
\frac{\bar{K}_{d}(\eta)}{k T}=\left(\frac{7}{4}\right) \frac{2 \sqrt{\eta_{e f f}}\left(3+2 \eta_{e f f}\right)-3 \sqrt{\pi} e^{\eta_{e f f}} \operatorname{erf}\left(\sqrt{\eta_{e f f}}\right)}{6 \sqrt{\eta_{e f f}}-3 \sqrt{\pi} e^{\eta_{e f f}} \operatorname{erf}\left(\sqrt{\eta_{e f f}}\right)} .
$$

The mean potential energy of collisionally lost atoms is given by

$$
\frac{\bar{U}_{d}(\eta)}{k T}=\frac{\int_{0}^{\chi} \eta \rho e^{-2 \eta \rho} \rho^{2} d \rho}{\int_{0}^{\chi} e^{-2 \eta \rho} \rho^{2} d \rho}=\frac{3}{2}+\frac{2(\chi \eta)^{3}}{1-e^{2 \chi \eta}+2 \chi \eta(1+\chi \eta)} .
$$

The mean energy of collisionally lost atoms is then given by the sum of the kinetic and potential energies,

$$
\bar{E}_{d}(\eta)=\bar{U}_{d}(\eta)+\bar{K}_{d}(\eta),
$$

and is plotted in Fig. 6 below.

The average evaporation energy is obtained by modeling the process as if all evaporation resulted from collisions occurring at the effective radius. The evaporation rate is proportional to the collisional velocity between atoms which scales like $\sqrt{\varepsilon}$. We model the process as if the evaporation takes place in a box potential with the threshold kinetic energy given by $\eta_{\text {eff. }}$ Using the free particle phase space density, proportional to $\sqrt{\varepsilon}$, we obtain

$$
\bar{K}_{v}(\eta)=\frac{\int_{\eta_{e f f}}^{\infty} \varepsilon^{2} e^{-\varepsilon} d \varepsilon}{\int_{\eta_{e f f}}^{\infty} \varepsilon e^{-\varepsilon} d \varepsilon}=\frac{\eta_{e f f}^{2}+2 \eta_{e f f}+2}{\eta_{e f f}+1} .
$$

To this average kinetic energy, we must add the potential energy at the effective radius where the evaporating collisions are assumed to occur. This gives

$$
\bar{E}_{v}(\eta)=K_{v}(\eta)+\eta \rho_{e f f}=6^{1 / 3}+\frac{\eta_{e f f}^{2}+2 \eta_{e f f}+2}{\eta_{e f f}+1},
$$

which is plotted in Fig. 7 below.

\section{EXACT SOLUTION FOR THE SPHERICAL TRAP}

As $\eta$ is reduced, the primary mechanism responsible for the failure of the large- $\eta$ approximation is the distortion of the atom distributions from their ideal Boltzmann shape. We now develop expressions for the distribution functions that remain valid for all $\eta$ in a linear spherical trap.

\section{A. Dimensionless coordinates for spherical trap}

It is useful to transform the phase space volume element $d^{3} r d^{3} p$, into coordinates appropriate for describing the spherically symmetric system $d^{3} r d^{3} p \rightarrow d r d \theta d \phi d p_{r} d L d L_{z}$, where $r, \theta$, and $\phi$ are the spherical polar coordinates, $p_{r}$ is the radial momentum, $L$ is the total angular momentum, and $L_{z}$ is its projection along the $z$ axis $(\theta=0)$. Jacobian transformations can be used to transform the phase space volume element into spherically symmetric coordinates, giving

$$
d^{3} r d^{3} p=\frac{L}{\sqrt{L^{2}-L_{z}^{2}}} d r d \theta d \phi d P_{r} d L d L_{z} .
$$

The conserved angular momentum imposed by the spherical symmetry of the trapping potential allows the energy of a trapped atom to be written as

$$
E=p_{r}^{2} /(2 m)+L^{2} /\left(2 m r^{2}\right)+G r,
$$

where $G r$ is the radially dependent potential energy. The motion of the radial coordinate can be described as if the atom were confined in the effective radial potential

$$
V_{L}(r)=L^{2} /\left(2 m r^{2}\right)+G r .
$$

In a spherical trapping potential, the maximum allowable energy and angular momentum are realized by placing a particle in a circular orbit at the radius of the trap wall, $R$. Defining the potential at the trap wall to be $U_{w} \equiv G R$, we can express the maximum energy and the maximum angular momentum in the trap as

$$
E_{m}=\frac{3}{2} U_{w}, L_{m}^{2}=m R^{2} U_{w} .
$$

We now scale the radius, angular momentum, and energy to define the dimensionless variables

$$
\rho \equiv \frac{r}{R}, £ \equiv \frac{L}{L_{m}}, Q \equiv \frac{E}{U_{w}} .
$$

\section{B. Allowed orbits}

For a given angular momentum, the effective potential Eq. (32) can be recast in dimensionless units as

$$
V_{£}(\rho)=\frac{£^{2}}{2 \rho^{2}}+\rho .
$$

A plot of this effective potential for several different values of $£$ is shown in Fig. 1. The lowermost curve (i.e., the straight line) is the effective potential for zero angular momentum. The uppermost curve is the effective potential for the highest angular momentum allowed in the trap (i.e., $£^{2}$ $=1$ ).

Consider all atoms with some energy $Q$ that pass through a given radius $\rho$. Let the energy of one of these atoms correspond to the dashed line in Fig. 1. For a given angular momentum, the classical turning points are given by the intersection of the dashed line with the corresponding effective potential curve. Increasing this angular momentum decreases the distance between the turning points until they meet to 


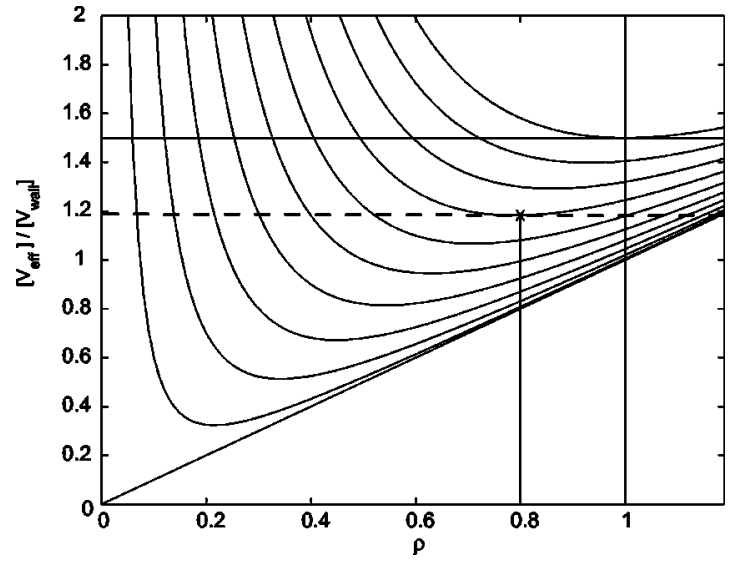

FIG. 1. Effective trapping potentials for atoms with angular momentum taking on values of $£=0,0.1, \ldots, 1$. The $x$ axis of this plot is the radial coordinate of the trap in units of the maximum trap radius. The $y$ axis is the effective potential (including angular momentum barrier) in units of the potential energy at the trap wall.

define a circular orbit (the cross in Fig. 1) at the maximum angular momentum value allowed for an atom having energy $Q$ and passing through radius $\rho$,

$$
£_{\max }^{2}(Q, \rho)=2 \rho^{2}(Q-\rho) .
$$

As the angular momentum is decreased from its maximum value, the outer turning point occurs at ever increasing radii until it intersects the trap wall. This condition defines the minimum angular momentum for trapped atoms having energy $Q$,

$$
£_{\text {min }}^{2}(Q)=2(Q-1)
$$

(subject to the constraint that $£_{\min }^{2} \geqslant 0$ ).

The minimum possible energy for an atom at radius $\rho$ is realized when its kinetic energy is zero,

$$
Q_{\min }(\rho)=\rho .
$$

The maximum possible energy for an atom passing through radius $\rho$ is realized by selecting the angular momentum that maximizes the kinetic energy. This happens for the angular momentum whose effective potential curve has turning points at both $\rho$ and the trap wall. The maximum energy on this potential curve is

$$
Q_{\max }(\rho)=1+\frac{\rho^{2}}{1+\rho} .
$$

The largest possible radius for atoms of energy $Q$ is realized at the outer turning point of the $£=0$ effective potential curve,

$$
\rho_{\max }(Q)=Q
$$

(subject to the constraint that $\rho_{\max } \leqslant 1$ ).

Figure 1 shows that atoms with $Q>1$ must have $£>0$. Due to the angular momentum barrier, no orbits with $£>0$ are allowed near the trap center. Therefore, atoms with energy greater than the trap depth will be confined to radii larger than the minimum value,

$$
\begin{gathered}
\rho_{\min }(Q \leqslant 1)=0 \\
\rho_{\text {min }}(Q>1)=\frac{1}{2}(Q-1)+\frac{1}{2} \sqrt{Q^{2}+2 Q-3} .
\end{gathered}
$$

\section{Density of states}

The density of states for a spherical linear trap is

$$
\begin{aligned}
g(E) d E & =d E \frac{1}{h^{3}} \iint \delta\left(E-G r-\frac{p^{2}}{2 m}\right) d^{3} r d^{3} p \\
& =d E\left(\frac{\pi}{h}\right)^{3} \sqrt{2 m} \iint \frac{d\left(L^{2}\right) d r}{\sqrt{E-L^{2} /\left(2 m r^{2}\right)-G r}} .
\end{aligned}
$$

Transforming to dimensionless variables and integrating over the allowed angular momentum, we obtain the density of trapped states

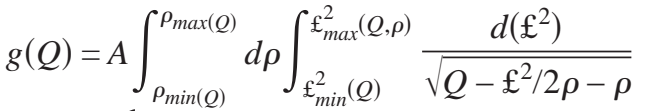

$$
\begin{aligned}
& = \begin{cases}\frac{64}{105} A Q^{7 / 2}, & Q \in[0,1], \\
4 A \int_{\rho_{\min (Q)}}^{\rho_{\max (Q)}} \sqrt{Q\left(\rho^{2}-1\right)-\left(\rho^{3}-1\right)} d \rho, & Q \in\left(1, \frac{3}{2}\right], \\
0, & Q \in\left(\frac{3}{2}, \infty\right],\end{cases}
\end{aligned}
$$

where $A \equiv(\pi / h)^{3} \sqrt{2(m \eta k T)^{3}}$. One can fit to an ad hoc analytic expression for the remaining integral in Eq. (43) and find

$g(Q)$

$$
\simeq \begin{cases}\frac{64}{105} A Q^{7 / 2}, & Q \in[0,1], \\ 70 A \exp \left[-4\left(\frac{3}{2}-Q\right)^{-1 / 4}-(Q-1)\right], & Q \in\left(1, \frac{3}{2}\right), \\ 0, & Q \in\left(\frac{3}{2}, \infty\right] .\end{cases}
$$

This ad hoc fit is quite useful in numerical calculations such as finding the evaporation fraction.

\section{Energy dependent density distribution}

From the expression for the density states Eq. (43), the probability that a particle with energy between $Q$ and $Q$ $+d Q$ is found between radii $\rho$ and $\rho+d \rho$ is (in a Boltzmann distribution) 

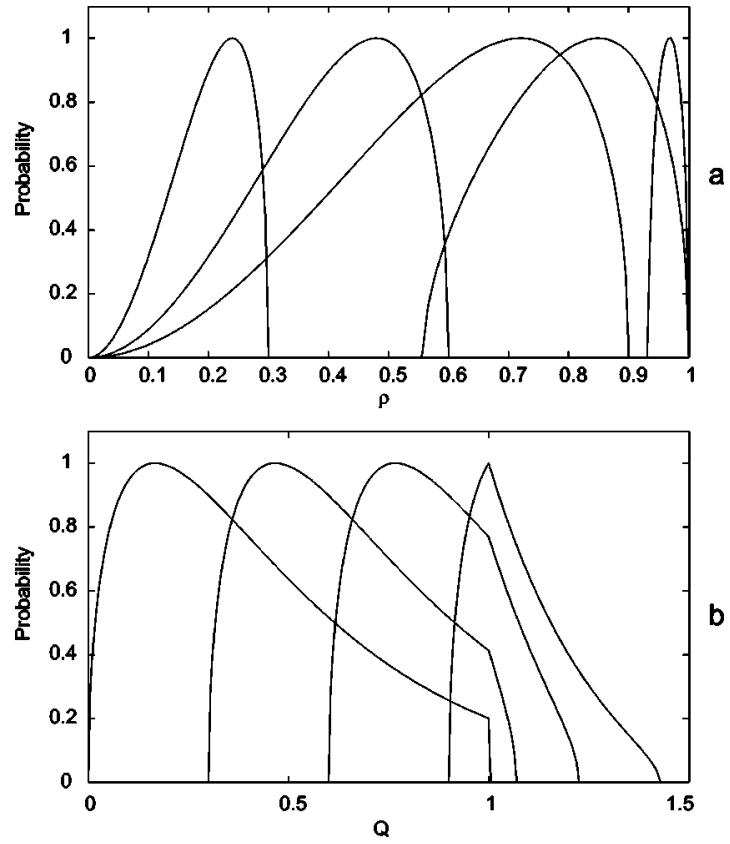

FIG. 2. (a) These five traces are the radial probability distributions for energies of $Q=0.3,0.6,0.9,1.2,1.45$. The distributions have been scaled to have a peak value of unity. (b) These four traces are the energy probability distributions for atoms found at radii $\rho$ $=0,0.3,0.6,0.9$. They have also been scaled to have peak values of unity.

$$
\begin{aligned}
& P(\rho, Q) d \rho d Q \\
& \propto d \rho d Q \exp (-\eta Q) \int_{\mathfrak{£}_{\min }^{2}(Q)}^{\mathfrak{f}_{\max }^{2}(Q, \rho)} \frac{d\left(£^{2}\right)}{\sqrt{Q-£^{2} / 2 \rho-\rho}} \\
& \propto \begin{cases}\rho \sqrt{Q\left(\rho^{2}-1\right)+\left(1-\rho^{3}\right)} \exp (-\eta Q) d Q, & Q>1, \\
\rho^{2} \sqrt{Q-\rho} \exp (-\eta Q) d Q, & Q \leqslant 1 .\end{cases}
\end{aligned}
$$

Equation (45) is graphically presented in Fig. 2. Each trace has been rescaled to have peak values of unity. Figure

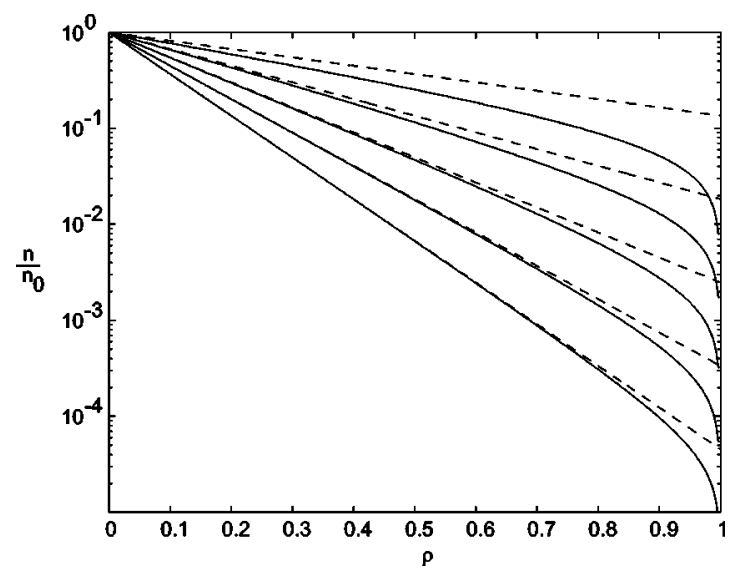

FIG. 3. The solid lines show the trapped density distributions obtained by numerically integrating Eq. (47) for $\eta=2,4,6,810$. The large- $\eta$ approximation for the density, $n=n_{0} e^{-\eta \rho}$, is shown by the dotted traces for the same values of $\eta$.

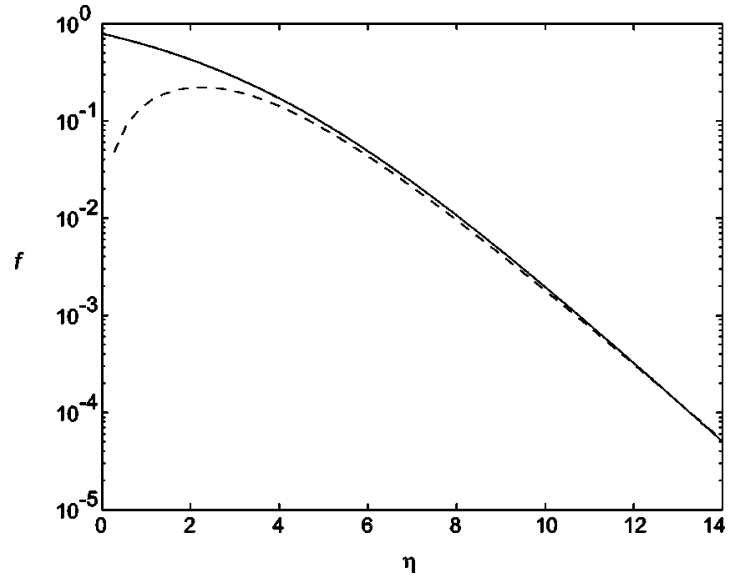

FIG. 4. The solid line is the result of numerically integrating Eq. (54) to obtain the fraction of elastic collisions that result in an evaporating atom. The dashed line is a plot of the large- $\eta$ approximation for the evaporation fraction given in Eq. (11).

2(a) shows the spatial distribution for atoms at a few representative energy values. Figure 2(b) shows the energy distribution of atoms passing through a few representative radii. Note that the energy distribution for atoms near the center of the trap is closely approximated by a Boltzmann distribution truncated at $Q=1$, which is equivalent to $E=\eta k T$. This is the usual truncation energy assumed in the large- $\eta$ approximation. Since most atoms will be pulled near the trap center at large $\eta$, the atoms' energy distribution will be most like the leftmost trace of Fig. 2(b) and the large- $\eta$ approximation is justified. At lower $\eta$, however, atoms will populate larger radii, thereby distorting the overall energy distribution and causing the large $\eta$ approximation to break down.

Finally, since the density varies as the probability divided by the differential volume, the energy-dependent density distribution is

$n(\rho, Q) d Q$

$$
\propto \begin{cases}\frac{1}{\rho} \sqrt{Q\left(\rho^{2}-1\right)+\left(1-\rho^{3}\right)} \exp (-\eta Q) d Q, & Q>1, \\ \sqrt{Q-\rho} \exp (-\eta Q) d Q, & Q \leqslant 1 .\end{cases}
$$

\section{E. Density distribution}

The density at any point in the trap is obtained by integrating Eq. (46) over the allowed energy values to get

$$
\begin{aligned}
n(\rho) & =n_{0} \frac{\left(\int_{Q_{\min }(\rho)}^{Q_{\max }(\rho)} n(\rho, Q) d Q\right)}{\left(\int_{0}^{1} n(0, Q) d Q\right)} \\
& =n_{0} \frac{\exp \left(x^{2}\right) \operatorname{erf}(x)-(x / y)(1 / i) \operatorname{erf}(i y) \exp \left(-y^{2}\right)}{\exp (\eta) \operatorname{erf}(\sqrt{\eta})-2 \sqrt{\eta / \pi}}
\end{aligned}
$$

where $n_{0}$ is the density at the center of the trap, $x$ 
$\equiv \sqrt{\eta(1-\rho)}$, and $y \equiv \sqrt{\eta \rho^{2} /(1+\rho)}$. Using a series expansion for the complex error function [11], we obtain

$$
n(\rho)=n_{0} \frac{\exp \left(x^{2}\right) \operatorname{erf}(x)-(x / y) S_{N}(y) \exp \left(-y^{2}\right)}{\exp (\eta) \operatorname{erf}(\sqrt{\eta})-2 \sqrt{\eta / \pi}},
$$

where $S_{N}(y) \equiv y / \pi+(2 / \pi) \sum_{n=1}^{N}\left(e^{-n^{2} / 4} n\right) \sinh (n y)$.

Figure 3 compares this density distribution with that obtained from the large- $\eta$ approximation, $n=n_{0} e^{-\eta \rho}$. As a rule of thumb, the large- $\eta$ approximation can be used for $\eta \geq 4$.

\section{F. Evaporation probability}

For an atom exiting a collision with energy $Q$, we postulate that the random nature of the scattering process places it into any energetically allowed trajectory with equal probability. The likelihood that this atom remains confined is then given by the fraction of these possible trajectories that do not intersect the trap wall. This fraction is identically the ratio of the density of states of a finite trap to that of an infinite trap. Thus the probability of evaporation is

$$
P_{v}(Q)=1-\frac{g(Q)}{g_{\infty}(Q)} .
$$

Using Eq. (44) for the density of states, we find

$$
P_{v}(Q) \simeq \begin{cases}0, & Q \in[0,1], \\ 1-\frac{3675}{32 Q^{7 / 2}} \exp \left[-4\left(\frac{3}{2}-Q\right)^{-1 / 4}-(Q-1)\right], & Q \in\left(1, \frac{3}{2}\right), \\ 1, & Q \in\left(\frac{3}{2}, \infty\right] .\end{cases}
$$

\section{G. Evaporation fraction}

The total collision rate for atoms in the differential volume about $\rho$ is

$$
\begin{aligned}
d \Gamma_{e l}(\rho) \propto & \sigma_{e l} \rho^{2} d \rho \int_{Q_{\min (\rho)}}^{Q_{\max }(\rho)} \int_{Q_{\min (\rho)}^{Q_{\max }(\rho)}} \\
& \times n\left(\rho, Q_{1}\right) v_{r e l}\left(Q_{1}, Q_{2}, \rho\right) n\left(\rho, Q_{2}\right) d Q_{2} d Q_{1}
\end{aligned}
$$

where $v_{\text {rel }}\left(Q_{1}, Q_{2}, \rho\right) \propto \sqrt{Q_{1}+Q_{2}-2 \rho}$ is the relative velocity.

The dimensionless kinetic energies are given by $\tau_{1}=Q_{1}$ $-\rho$ and $\tau_{2}=Q_{2}-\rho$, respectively. Assuming that each elastic collision randomizes the scattered velocity directions in the center of mass frame, it can be shown that for two colliding atoms with total lab-frame kinetic energy $\tau=\tau_{1}+\tau_{2}$, the probability that one atom exits the collision having kinetic energy (in the lab frame) between $H_{1}$ and $H_{1}+d H_{1}$ is

$$
P_{s}\left(\tau, H_{1}\right) d H_{1}=\frac{2}{\tau} \sin ^{-1}\left(\frac{2 \sqrt{H_{1}\left(\tau-H_{1}\right)}}{\tau}\right) d H_{1} .
$$

The probability that this atom evaporates is then given by $P_{v}\left(\rho+H_{1}\right)$, where $\rho+H_{1}$ is its total energy. This allows us to write the evaporation rate of the atoms found in the differential volume about $\rho$,

$$
\begin{aligned}
d \Gamma_{\text {evap }}(\rho)= & \sigma_{e l} \rho^{2} d \rho \int_{Q_{\min (\rho)}^{Q_{\max }(\rho)}}^{Q_{Q_{\min }(\rho)}(\rho)} n\left(\rho, Q_{1}\right) v_{r e l}\left(Q_{1}, Q_{2}, \rho\right) \\
& \times n\left(\rho, Q_{2}\right) \int_{0}^{\tau} P_{s}\left(\tau, H_{1}\right) P_{v}\left(\rho+H_{1}\right) d H_{1} d Q_{2} d Q_{1} .
\end{aligned}
$$

Integrating over the volume of the trap and dividing by the total elastic collision rate, we obtain an expression for the fraction of elastic collisions that result in an evaporating atom,

$$
f(\eta)=\frac{\int_{0}^{1} \rho^{2} d \rho\left(\int_{Q_{\min }}^{Q_{\max }} n\left(\rho, Q_{1}\right) d Q_{1} \int_{Q_{\min }}^{Q_{\max }} v_{r e l}\left(Q_{1}, Q_{2}, \rho\right) n\left(\rho, Q_{2}\right) d Q_{2} 2 \int_{\tau / 2}^{\tau} P_{s}\left(\tau, H_{1}\right) P_{v}\left(\rho+H_{1}\right) d H_{1}\right)}{\int_{0}^{1} \rho^{2} d \rho\left(\int_{Q_{\min }}^{Q_{\max }} n\left(\rho, Q_{1}\right) d Q_{1} \int_{Q_{\min }}^{Q_{\max }} v_{r e l}\left(Q_{1}, Q_{2}, \rho\right) n\left(\rho, Q_{2}\right) d Q_{2}\right)} .
$$




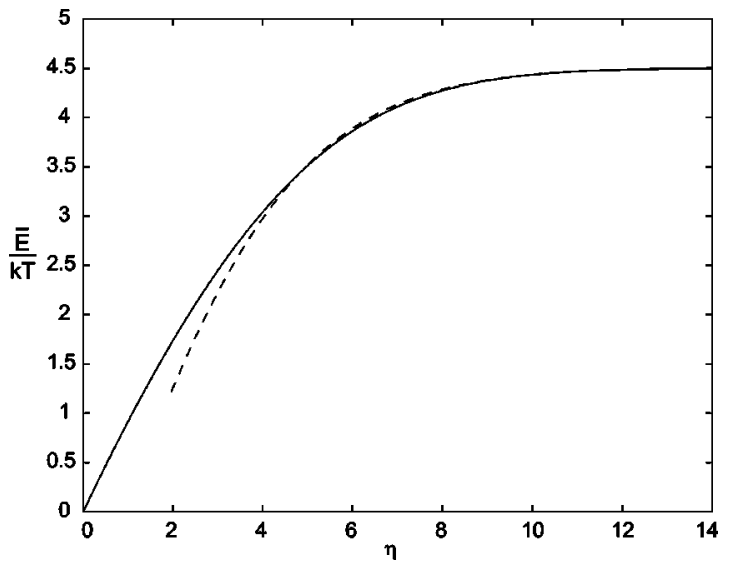

FIG. 5. The solid line shows the $\eta$ dependence of the average energy of a trapped atom, $\bar{E} / k T$, as given by numerically integrating Eq. (55). The dashed line is a plot of the large- $\eta$ approximation as given by Eq. (22).

Figure 4 compares this evaporation fraction with that obtained in the large- $\eta$ approximation. As before, the large- $\eta$ approximation gives good agreement for $\eta \gtrsim 4$.

\section{H. Average energies and effective volumes}

The average energy in the trap is straightforward to calculate. Using $n(\rho, Q)$ from Eq. (46), the mean energy is simply

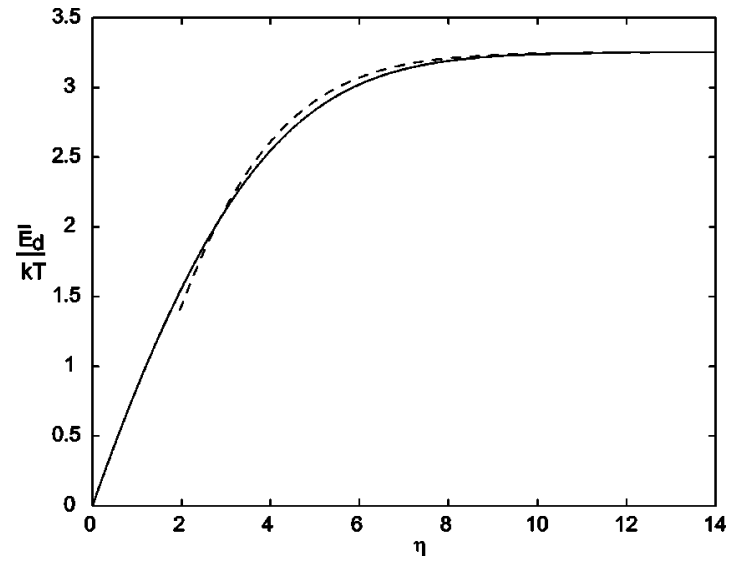

FIG. 6. The solid curve shows the $\eta$ dependence of the average energy of atoms lost due to inelastic collisions, $\bar{E}_{d} / k T$, as given by numerically integrating Eq. (56). The dashed curve is a plot of the large- $\eta$ approximation as given by Eq. (27).

$$
\bar{Q}(\eta)=\frac{\int_{0}^{1} \rho^{2} d \rho \int_{Q_{\min }}^{Q_{\max }} Q n(\rho, Q) d Q}{\int_{0}^{1} \rho^{2} d \rho \int_{Q_{\min }}^{Q_{\max }} n(\rho, Q) d Q}
$$

where $Q_{\min }=\rho$ and $Q_{\max }=1+\rho^{2} /(1+\rho)$. Figure 5 shows a comparison of the average energy in the trap compared with the result of the large- $\eta$ approximation.

Applying Eq. (51) to describe inelastic collisions, the average energy of atoms lost due to inelastic processes can be written as

$$
\bar{Q}_{d}(\eta)=\frac{\int_{0}^{1} \rho^{2} d \rho \int_{Q_{\min }}^{Q_{\max }} Q_{1} n\left(\rho, Q_{1}\right) d Q_{1} \int_{Q_{\min }}^{Q_{\max }} v_{r e l}\left(Q_{1}, Q_{2}, \rho\right) n\left(\rho, Q_{2}\right) d Q_{2}}{\int_{0}^{Q_{\max }} \rho^{2} d \rho \int_{Q_{\min }}^{Q_{\max }} n\left(\rho, Q_{1}\right) d Q_{1} \int_{Q_{\min }} v_{r e l}\left(Q_{1}, Q_{2}, \rho\right) n\left(\rho, Q_{2}\right) d Q_{2}} .
$$

Figure 6 shows a comparison of the average energy removed by inelastic collisions compared with the results obtained in the large- $\eta$ approximation.

The average energy of an evaporating atom is calculated by multiplying the differential evaporation rate by the energy an evaporating atom removes, $\left(\rho+H_{1}\right)$. Integrating and normalizing by the evaporation rate gives

$$
\bar{Q}_{v}(\eta)=\frac{\int_{0}^{1} \rho^{2} d \rho\left[\int_{Q_{\min }}^{Q_{\max }} n\left(\rho, Q_{1}\right) d Q_{1} \int_{Q_{\min }}^{Q_{\max }} v_{r e l}\left(Q_{1}, Q_{2}, \rho\right) n\left(\rho, Q_{2}\right) d Q_{2} \int_{\tau / 2}^{\tau}\left(\rho+H_{1}\right) P_{s}\left(\tau, H_{1}\right) P_{v}\left(\rho+H_{1}\right) d H_{1}\right]}{\int_{0}^{1} \rho^{2} d \rho\left[\int_{Q_{\min }}^{Q_{\max }} n\left(\rho, Q_{1}\right) d Q_{1} \int_{Q_{\min }}^{Q_{\max }} v_{r e l}\left(Q_{1}, Q_{2}, \rho\right) n\left(\rho, Q_{2}\right) d Q_{2} \int_{\tau / 2}^{\tau} P_{s}\left(\tau, H_{1}\right) P_{v}\left(\rho+H_{1}\right) d H_{1}\right]} .
$$




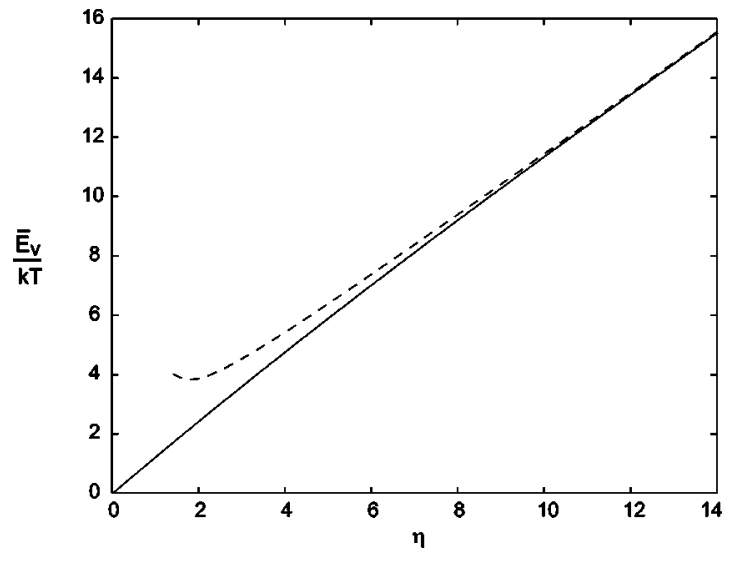

FIG. 7. The solid line shows the $\eta$ dependence of the average energy of an evaporated atom, $\bar{E}_{v} / k T$, as given by numerically integrating Eq. (57). The dashed line is the large- $\eta$ approximation given by Eq. (29).

Figure 7 shows the average energy removed by evaporating atom compared to the result of the large- $\eta$ approximation.

The effective volumes can be calculated using the same method taken in the large- $\eta$ approximation, but using Eq. (48) for the density:

$$
\begin{gathered}
V_{e f f}(\eta)=3 V_{0} \int_{0}^{1} \frac{n(\rho)}{n_{0}} \rho^{2} d \rho, \\
\Lambda_{e f f}(\eta)=3 V_{0} \int_{0}^{1}\left[\frac{n(\rho)}{n_{0}}\right]^{2} \rho^{2} d \rho .
\end{gathered}
$$

\section{Equilibrium $\boldsymbol{\eta}$ and cross-section ratio}

In sufficiently good vacuum, the competition between evaporative cooling and inelastic collisional heating drives the atoms to a thermal distribution with a steady state temperature. The ratio of the trap depth to this steady state temperature is defined to be the equilibrium $\eta$.

We now show that the equilibrium $\eta$ depends only on the ratio of elastic to inelastic cross sections, $\gamma \equiv \sigma_{e l} / \sigma_{i n}$. Time differentiating the total energy in the trap, $E=N \bar{E}$, and noting that the time derivative of the average energy vanishes for constant temperature, we obtain

$$
\bar{E}=\frac{\dot{E}}{\dot{N}}=\frac{\dot{N}_{v} \bar{E}_{v}+\dot{N}_{d} \bar{E}_{d}}{\dot{N}_{v}+\dot{N}_{d}}=\frac{f \gamma \bar{E}_{v}+\bar{E}_{d}}{f \gamma+1},
$$

where $\dot{N}_{v}$ is the evaporative loss rate, $\dot{N}_{d}$ is the inelastic loss rate, and $f$ is the fraction of elastic collisions that produce an evaporating atom. Dividing through by $\eta k T$ and solving for $\gamma$, we obtain

$$
\gamma \equiv \frac{\sigma_{e l}}{\sigma_{i n}}=\frac{\bar{Q}-\bar{Q}_{d}}{f\left(\bar{Q}_{v}-\bar{Q}\right)} .
$$

Each variable on the right-hand side of Eq. (60) depends only on $\eta$, showing that the cross-section ratio uniquely de-

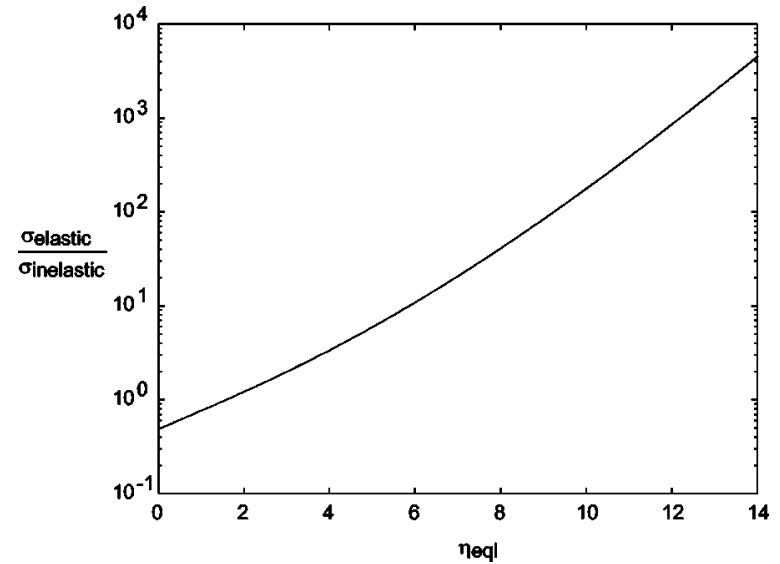

FIG. 8. The ratio between the elastic and inelastic scattering rates causes the temperature of the trapped atoms to approach a fixed fraction of the trap depth. This figure shows the cross section ratio that must exist to produce a given "equilibrium $\eta$." The solid curve is the result of the exact treatment. The dashed curve is the result of the large- $\eta$ approximation.

termines the equilibrium $\eta$. This dependence is illustrated in Fig. 8.

\section{J. Collision rate coefficients}

Assuming that each inelastic collision results in one atom being ejected from the trap, the inelastic loss rate is

$$
\dot{N}_{i n}=\Lambda_{e f f} \sigma(\bar{v} \sqrt{2}) n_{0}^{2}=\Lambda_{e f f}(\eta) g_{i n} n_{0}^{2} .
$$

For a trapped ensemble at constant temperature, $\dot{N}$ $=V_{\text {eff }}(\eta) \dot{n}_{0}$. Using this, we obtain a differential equation for the peak density in the trap when only inelastic losses are considered:

$$
\left[\dot{n}_{0}\right]_{i n}=-g_{\text {in }} \frac{\Lambda_{\text {eff }}}{V_{\text {eff }}} n_{0}^{2} .
$$

Using a similar analysis for the evaporation process, the differential equation for the peak density in the trap due to both evaporation and inelastic collisions is

$$
\dot{n}_{0}=-\left(g_{\text {in }}+f g_{\text {el }}\right) \frac{\Lambda_{\text {eff }}}{V_{e f f}} n_{0}^{2}=-g_{2 b} n_{0}^{2},
$$

where $g_{e l}$ and $g_{\text {in }}$ are the elastic and inelastic scattering rate coefficients, $f$ is the evaporation fraction, and $g_{2 b}$ $\equiv\left(\Lambda_{e f f} / V_{e f f}\right)\left(g_{\text {in }}+f g_{e l}\right)$. Since $g_{2 b}$ and $\eta$ are the usual experimentally measurable quantities, the elastic and inelastic scattering rate coefficients can be expressed in terms of them as

$$
\begin{aligned}
& g_{\text {in }}=\frac{V_{e f f}}{\Lambda_{e f f}} \frac{1}{f \gamma+1} g_{2 b}, \\
& g_{e l}=\frac{V_{e f f}}{\Lambda_{e f f}} \frac{\gamma}{f \gamma+1} g_{2 b} .
\end{aligned}
$$




\section{CONCLUSIONS}

The literature contains useful models for understanding trapped atomic gases in the high- $\eta$ limit. At low $\eta$, however, these models are not applicable and lack straightforward analytical expressions for the relevant distribution functions, average energies, evaporation fraction, etc. In this paper, we have derived useful analytic expressions that describe ensembles of atoms confined at arbitrary $\eta$ in a linear spherical trapping potential. The features of this spherical model pro- vide intuitive insight into the dynamics of evaporation and collisional loss at arbitrary $\eta$.

Although this model is constructed for the special case of a linear spherical trapping potential, the approach of transforming phase space variables to the appropriate coordinate system, determining orbital constraints, and integrating over the Boltzmann distribution is general. It can be applied to describe atoms confined in any trap shape (e.g., spherical parabolic, linear cylindrical, elliptical, etc.)
[1] J. M. Doyle, B. Friedrich, J. Kim, and D. Patterson, Phys. Rev. A 52, R2515 (1995).

[2] J. Kim, B. Friedrich, D. P. Katz, D. Patterson, J. D. Weinstein, R. deCarvalho, and J. M. Doyle, Phys. Rev. Lett. 78, 3665 (1997).

[3] J. D. Weinstein, R. deCarvalho, J. Kim, D. Patterson, B. Friedrich, and J. M. Doyle, Phys. Rev. A 57, R3173 (1998).

[4] J. D. Weinstein, R. deCarvalho, T. Guillet, B. Friedrich, and J. M. Doyle, Nature (London) 395, 148 (1998).

[5] R. deCarvalho, J. M. Doyle, B. Friedrich, T. Guillet, J. Kim, D. Patterson, and J. D. Weinstein, Eur. Phys. J. D 7, 289 (1999).

[6] J. D. Weinstein, R. deCarvalho, C. I. Hancox, and J. M. Doyle,
Phys. Rev. A 65, 021604 (2002).

[7] R. deCarvalho, C. I. Hancox, and J. M. Doyle, J. Opt. Soc. Am. B 20, 1131 (2003).

[8] O. J. Luiten, M. W. Reynolds, and J. T. M. Walraven, Phys. Rev. A 53, 381 (1996).

[9] J. M. Doyle, Ph.D. thesis, MIT, 1991.

[10] W. Ketterle and N. V. Druten, Adv. At., Mol., Opt. Phys. 37, 181 (1996).

[11] Handbook of Mathemetical Functions With Formulas, Graphs, and Mathematical Tables, Natl. Bur. Stand. Appl. Math. Ser. No. 55, edited by M. Abramowitz and I. A. Stegun (U.S. GPO, Washington, D.C., 1965). 